\title{
Analysis of TNF-like weak inducer of apoptosis for detecting lupus nephritis
}

\author{
Hani Susianti ${ }^{1}$ - Dian Sukma Hanggara ${ }^{1} \cdot$ Kristina Dyah Lestari $^{2} \cdot$ Putri Purnamasari $^{2} \cdot$ Andrea Aprilia $^{1}$ (D
}

Received: 8 November 2021 / Accepted: 23 February 2022 / Published online: 28 February 2022

(c) The Author(s), under exclusive licence to Springer-Verlag London Ltd., part of Springer Nature 2022

\begin{abstract}
Lupus is an autoimmune disease that has various manifestations in various organs. One of the manifestations of lupus is lupus nephritis (LN), which often causes kidney failure and death. Cytokines play an essential role in the pathogenesis of LN and might be helpful for LN biomarkers. This study aimed to evaluate urine TNF-like weak inducer of apoptosis (TWEAK) for detecting LN since this is not an invasive procedure and is more cost-effective. The gold standard procedure for diagnosing LN needs a biopsy of the kidney. However, the procedure is invasive, high cost, and takes time. Thus, a biomarker from urine is needed for early diagnosis of LN. This research conducted was cross-sectional. The total participants were 57, consisting of 29 lupus nephritis and 28 lupus without nephritis. TWEAK levels were determined by ELISA method; urine protein, urine erythrocyte, and leukocyte were examined by a urine autoanalyzer. Statistical analysis using Mann-Whitney, Spearman correlation, Kruskal-Wallis, ROC curve analysis, and a $2 \times 2$ contingency table. This study showed a significant difference in TWEAK levels between lupus nephritis and lupus without nephritis $(p<0.05)$, but no significant difference between TWEAK level and renal domain scores of SLEDAI. There were significant correlations between TWEAK level and urine erythrocyte and urine protein, but there was no significant correlation with urine leukocytes. The sensitivity and specificity of TWEAK for determining LN were $72.4 \%$ and $72.5 \%$, respectively, with AUC 0.77 . TWEAK had a good diagnostic test for detecting lupus nephritis and substantially correlated with urine erythrocyte and urine protein.
\end{abstract}

Keywords Detecting $\cdot$ Lupus nephritis $\cdot$ TWEAK $\cdot$ Urine biomarker

\section{Introduction}

Lupus is an autoimmune disease that has various manifestations in various organs. One of the manifestations of lupus is lupus nephritis (LN), which occurs in about $60 \%$ of lupus patients and often results in kidney failure and death. This condition requires $\mathrm{LN}$ early diagnosis to make treatment faster and the response to therapy more profitable (Wu et al. 2018).

The complex etiopathogenesis and heterogeneous clinical manifestations make lupus often misdiagnosed. Identifying early $\mathrm{LN}$ biomarkers helps assess the risk of organ

Andrea Aprilia

andreaaprilia134@gmail.com

1 Clinical Pathology Department of Faculty Medicine Universitas Brawijaya/Dr, Saiful Anwar General Hospital, Malang, Indonesia

2 Department of Faculty Medicine Universitas Brawijaya/Dr, Saiful Anwar General Hospital, Malang, Indonesia involvement, predict the clinical manifestations that will develop, and provide the most appropriate therapy. So, it requires an early and accurate biomarker of $\mathrm{LN}$ diagnosis for better disease management (Stypińska and ParadowskaGorycka 2015; Reyes-Thomas et al. 2011).

TWEAK is a multifunctional protein and a member of the TNF cytokine superfamily (TNFSF). TWEAK is synthesized as a 249 -amino acid protein consisting of a C-terminal extracellular domain, a transmembrane domain, and an N-terminal intracellular domain. TWEAK binds to the Fn14 receptor (fibroblast growth factor-inducible 14), a type I transmembrane protein and is a member of the TNF receptor superfamily (TNFRSF). The TWEAK-Fn 14 axis regulates various physiological responses, including cell survival, proliferation, differentiation, angiogenesis, migration, and apoptosis. However, aberrant expression of TWEAK and Fn14 is associated with harmful pathogenic effects in autoimmune disorders, kidney damage, and dysfunction (Khairwa 2018; Bekhit et al. 2018). 
TWEAK also has a role as a proinflammatory molecule that plays a vital role in the active inflammation of lupus nephritis (Xuejing et al. 2012). TWEAK is predicted to be a biomarker for the early detection of LN. The currently existing LN biomarkers such as complement and anti-dsDNA are considered less sensitive or specific. Almost half of LN patients who clinically respond well to therapy still have active lesions when a kidney biopsy is performed (Lu et al. 2012; Suttichet et al. 2019). Based on the data above, there is an opportunity to examine TWEAK levels that may be used as biomarkers to early detect lupus nephritis.

\section{Method}

We conducted a cross-sectional study. The inclusion criteria were patients with lupus, based on the SLICC criteria, female, age 18 to 65 years old. The exclusion criteria were suffering from active infectious disease, diabetes, or kidney failure. The requirements of lupus nephritis were lupus patients with persistent proteinuria or greater than $0.5 \mathrm{~g} / \mathrm{day}$ (or on dipstick examination $3+$ ) or urine sediment abnormalities, or the kidney biopsy result indicated the lupus nephritis.

The urine samples were obtained by collecting urine from the midstream method to examine TWEAK levels using ELISA Kit. This study used TWEAK Thermo Fisher Scientific Inc. ELISA kit with catalogue number: BMS2006INNST, analytical sensitivity $9.7 \mathrm{pg} / \mathrm{mL}$, assay range $15.6-1,000 \mathrm{pg} / \mathrm{mL}$, interassay CV $9.2 \%$, and intraassay CV 7.9\%. Briefly, the well of the ELISA kit coated with anti-human TWEAK antibody was incubated with a diluted urine sample. After incubation for $2 \mathrm{~h}$ at room temperature, a peroxide-conjugated polyclonal anti-human TWEAK antibody and substrate were added. The reaction was stopped by adding acid, then read with a spectrophotometer at $450 \mathrm{~nm}$.
The results obtained were then plotted on a standard curve to obtain urine TWEAK levels.

The data were analyzed using SPSS version 18.0 software. The normality data was carried out using the Kolmogorov-Smirnov. Mann-Whitney tested differences in urine biomarker levels in the two groups. $P$-value $<0.05$ indicated a significant difference. Spearman's test analyzed the correlation of TWEAK with leukocyturia and erythrocyturia, and the correlation was significant if the $p$-value $<0.05$.The comparison of the TWEAK level in renal domain scores of SLEDAI (Systemic Lupus Erythematosus Disease Activity Index) were analyzed by the Kruskal-Wallis test. We used ROC curve analysis and a $2 \times 2$ contingency table for diagnostic test of TWEAK level in LN. The ethical committee approved this study of Saiful Anwar General Hospital, Malang, Indonesia (ethical number 400/247/K.3/302/2020). This study was approved by the ethical committee of Saiful Anwar General Hospital, Malang, Indonesia (ethical number 400/247/K.3/302/2020). All the patients included in this study were asked to sign an informed consent.

\section{Result}

A total of 57 study subjects consisted of 29 lupus nephritis and 28 lupus patients without nephritis. Subjects aged 18-70 years old, and the results of statistical tests stated that there was no significant difference in age between $\mathrm{LN}$ and lupus without nephritis participants, with $p$-value $=0.257$ (Table 1). The gender of all research subjects was female because hormonal factors influence the pathogenesis of lupus, so the gender of all research subjects is made the same.

This study showed that the urine TWEAK levels of patients in the lupus nephritis group increased significantly compared to those in the lupus group $(p<0.05)$. Table 2
Table 1 Characteristics data of participants

\begin{tabular}{|c|c|c|c|}
\hline Parameters & Lupus $(n=28)$ & Lupus nephritis $(\mathrm{n}=29)$ & $p$-value \\
\hline $\begin{array}{l}\text { Age (year) } \\
\text { (median, range) }\end{array}$ & $25(22-33)$ & $28(24-30)$ & 0.257 \\
\hline $\begin{array}{l}\text { Hemoglobin }(\mathrm{g} / \mathrm{dL}) \\
\text { (median, range) }\end{array}$ & $\begin{array}{l}12.05 \\
(11.33-12.77)\end{array}$ & $\begin{array}{l}11.62 \\
(10.87-12.36)\end{array}$ & 0.273 \\
\hline $\begin{array}{l}\text { Leukocyte count }\left(\times 10^{3} / \mathrm{uL}\right) \\
\text { (median, range) }\end{array}$ & $\begin{array}{l}6.950 \\
(5.999-7.902)\end{array}$ & $\begin{array}{l}8.562 \\
(7.114-10.010)\end{array}$ & 0.045 \\
\hline $\begin{array}{l}\text { Thrombocyte count }\left(\times 10^{3} / \mathrm{uL}\right) \\
\text { (Median, range) }\end{array}$ & $\begin{array}{l}248.821 \\
(205.324-292.318)\end{array}$ & $\begin{array}{l}272.000 \\
(239.002-304.997)\end{array}$ & 0.270 \\
\hline $\begin{array}{l}\text { Urine erythrocyte (/HPF) } \\
\text { (median, range) }\end{array}$ & $\begin{array}{l}4.40 \\
(0.86-9.66)\end{array}$ & $\begin{array}{l}22.61 \\
(-0.93-46.15)\end{array}$ & 0.001 \\
\hline $\begin{array}{l}\text { Urine leukocyte (/HPF) } \\
\text { (median, range) }\end{array}$ & $\begin{array}{l}9.07 \\
(4.27-22.42)\end{array}$ & $\begin{array}{l}13.71 \\
(4.35-23.08)\end{array}$ & 0.008 \\
\hline $\begin{array}{l}\text { Urine TWEAK (pg/mL) } \\
\text { (median, range) }\end{array}$ & $\begin{array}{l}22.4 \\
(13.1-31.8)\end{array}$ & $\begin{array}{l}102.9 \\
(35.5-170.3)\end{array}$ & 0.005 \\
\hline
\end{tabular}


Table 2 Correlation between TWEAK Levels and Urinalysis Examination

\begin{tabular}{llll}
\hline & $\begin{array}{l}\text { Urine } \\
\text { erythrocyte }\end{array}$ & Urine leukocyte & Urine protein \\
\hline TWEAK Level & $r=0.385$ & $r=0.073$ & $r=0.536$ \\
& $p=0.003$ & $p=0.594$ & $p=0.001$ \\
\hline
\end{tabular}

indicates the correlation between TWEAK with urine protein, erythrocyte, and leukocyte. We assessed the flare status of lupus nephritis patients in this study by adding up the SLEDAI (Systemic Lupus Erythematosus Disease Activity Index) scores in the renal domain, namely the presence of urine casts, urine erythrocytes, proteinuria, and urine leukocytes. According to the SLEDAI score, the highest score for the renal domain was 16. SLEDAI scores for the renal domain 4, 8, and 12 were obtained in 10, 8, and 11 patients, respectively (Table 3 ). The diagnostic performance (ROC, sensitivity value, specificity, cut-off point value) urine TWEAK for detecting LN is listed in Fig. 1. The ROC curve of the urine TWEAK levels for detecting lupus nephritis had an AUC of 0.77. Urine TWEAK had a sensitivity value of $72.4 \%$, specificity of $72.5 \%$, PPV of $65.6 \%$, and NPV of $78.4 \%$.

\section{Discussion}

Our study showed that the age range of non-nephritis lupus patients was 22-33 years old, and in nephritis lupus 24-30 years old; there was no significant difference between the ages of two groups. Many studies state that lupus often occurs in women aged 15-50 years. Rees et al. (2017) mentioned that the peak lifespan of lupus patients ranges from the third to the seventh decade of life in women. The age variations in some studies may be due to differences in research methods, diagnostic criteria, access to health facilities, access to immunological laboratory examinations, and different cut-offs for positive laboratory results, etc. (Rees et al. 2017).

Our study obtained that the urine TWEAK levels of the lupus nephritis (LN) increased significantly compared to those in the non-nephritis lupus group $(p<0.05)$. These results are similar to those shown by Schwartz et al. that the uTWEAK levels in the LN patient and the control group had

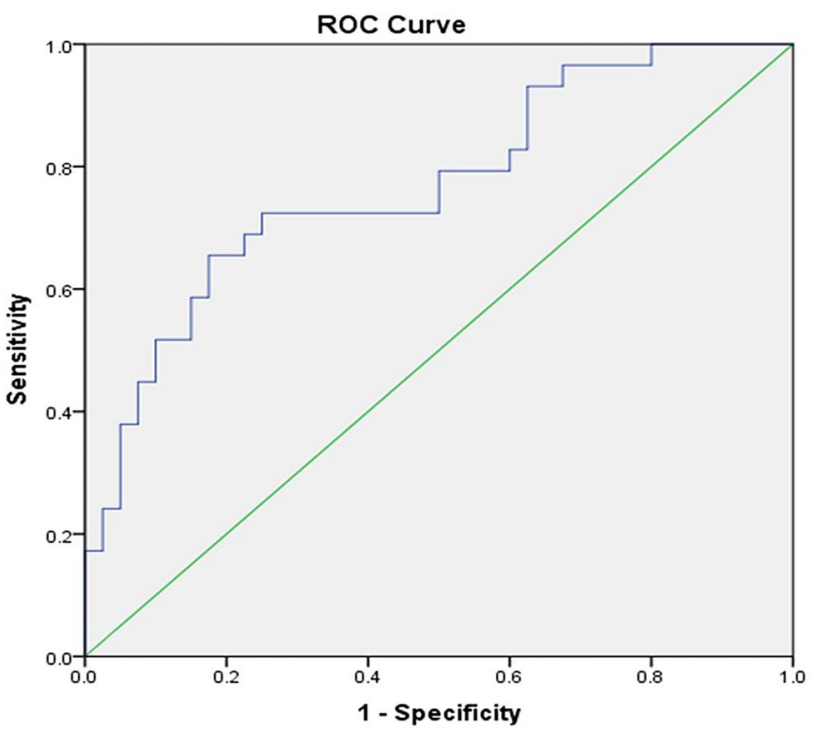

Fig. 1 ROC curve of urine TWEAK levels

a significant difference $(p=0.039)$. In addition, Schwartz et al. in the USA found that high uTWEAK levels were a specific characteristic of LN and not a general feature of SLE because the uTWEAK levels of non-nephritis lupus patients were not significantly different from the controls (Schwartz et al. 2009). The urine TWEAK levels in the lupus nephritis and the lupus group in our study are similar to studies conducted by Xuejing et al. (2012) and Sun et al. (2018). In our research, lupus nephritis patients had significantly higher urinary TWEAK levels than the lupus group. The results of our study and others support the statement that TWEAK plays an important role also in lupus nephritis occurring in Asia (Xuejing et al. 2012; Sun et al. 2018).

The results of our study showed that there were significant correlations between the TWEAK levels with urine erythroctyte and urine protein; however, there was no significant difference of the TWEAK level with the renal domain scores of SLEDAI (Systemic Lupus Erythematosus Disease Activity Index). The clinical manifestations of $\mathrm{LN}$ vary from asymptomatic urinalysis to nephrotic syndrome or medical emergencies (such as rapidly progressive glomerulonephritis syndrome). Symptoms of LN usually correlate with the degree of glomerular involvement (Almaani et al. 2017). In addition, the widely accepted criteria for the diagnosis of LN by the American College of Rheumatology (ACR) stated that kidney disorders in LN will show persistent proteinuria

Table 3 Comparison of TWEAK levels in renal domain scores of SLEDAI

\begin{tabular}{|c|c|c|c|c|}
\hline \multirow[t]{2}{*}{ Parameter } & \multicolumn{3}{|c|}{ Renal domain scores of SLEDAI } & \multirow[t]{2}{*}{$p$-value } \\
\hline & Score $4(n=10)$ & Score $8(n=8)$ & Score $12(n=12)$ & \\
\hline Urine TWEAK (pg/mL) (median, range) & $36.20(9.01-229.09)$ & $13.32(12.35-85.76)$ & $68.72(12.10-343.99)$ & 0.074 \\
\hline
\end{tabular}


(greater than $0.5 \mathrm{~g} /$ day) or urine sediment abnormalities such as an increase in red blood cells, hemoglobin, granular cast, and renal tubular cells (Farah et al. 2019; Hahn 2018). The significant correlation between the TWEAK levels with urine erythrocytes and proteinuria in our study suggests a role for TWEAK in the pathogenesis of LN; however, the TWEAK levels do not have an association with the disease activity of LN.

The role of TWEAK in LN pathogenesis induces the expression of several inflammatory mediators, including RANTES, monocyte chemoattractant protein (MCP)-1, IP-10, MIP-1 $\alpha$, ICAM-1, VCAM-1, MMP-1, and MMP-9. TWEAK-stimulated renal mesangial cells induce migration of peripheral blood mononuclear cells. In addition, TWEAK significantly stimulated the proliferation of renal mesangial and podocyte cells. TWEAK binding to its receptor (Fn14) can activate Iк-B and increase the expression of MCP-1, RANTES, and IL-6, causing inflammatory cell infiltration. The investigators also reported that TWEAK/Fn14 induces apoptosis of glomerular mesangial cells and tubular epithelial cells, thereby causing glomerular and tubular injury (Khairwa 2018; Salem et al. 2018).

Our study showed that urine TWEAK had a sensitivity value of $72.4 \%$ and specificity of $72.5 \%$, with PPV $65.6 \%$ and NPV $78.4 \%$ for detecting LN. These results are consistent with a meta-analysis by Lee and Song, which showed the sensitivity and specificity of urine TWEAK for the diagnosis of LN were $81.3 \%$ and $76.0 \%$, respectively, and the AUC of urinary TWEAK was 0.836 . These meta-analytical data suggest that urine TWEAK may be a valuable potential biomarker for assessing LN activity and diagnosis (Lee and Song 2017).

Our study's limitation was the small number of participants. It was challenging to get lupus patients in the COVID19 pandemic. Further studies need to be carried out by considering differences in LN activity and longitudinally to evaluate biomarkers with multiple measurements.

The present study found that TWEAK had better diagnostic testing for detecting lupus nephritis. There is a significant correlation between the TWEAK level with erythrocyte urine and protein urine.

Acknowledgements The authors thank all the participants in this study. The authors were thanking all the medical laboratory technicians in the Central Laboratory of Saiful Anwar General Hospital, especially those in the biomolecular and body fluid division for the cooperation during the conduction of this research.

Author contribution All the authors have contributed to all the processes in this research, along with the preparation, drafting, reviewing and approval of this manuscript.

Funding Research Institute and Community Service of Medicine Faculty, Brawijaya University funded this study.

\section{Declarations}

Conflict of interest The authors declare no competing interests.

\section{References}

Almaani S, Meara A, Rovin BH (2017) Update on lupus nephritis. Clin J Am Soc Nephrol 12:825-835

Bekhit MR, Kamel NS, Mohammed MO, Abaza NM, el Din SAS (2018) Role of tumor necrosis factor-like weak inducer of apoptosis/fibroblast growth factor-inducible molecule 14 pathway in lupus nephritis. Egypt Rheumatol Rehabil 45:182-187

Farah RI, Dannoun E, Abu Shahin N, Alryalat SA (2019) Characteristics and histological types of lupus nephritis in a Jordanian tertiary medical center. Biomed Res Int

Hahn B (2018) Systemic lupus erythematosus. In: Kasper DL, Fauci AS, Hauser SL, Longo DL, Jameson JL, Loscalzo J (eds) Harrison's Principles of internal medicine 20th edn. New York, McGraw Hill Professional

Khairwa A (2018) Role of urinary biomarkers for diagnosis of lupus nephritis. J Clin Exp Pathol 8:352-361

Lee YH, Song GG (2017) Urinary tumor necrosis factor-like weak inducer of apoptosis as a biomarker for lupus nephritis: a metaanalysis. J Rheum Dis 24:85-92

Lu J, Kwan BCH, Lai FMM, Tam LS, Li EKM, Chow KM, Wang G, Li PKT, Szeto CC (2012) Glomerular and tubulointerstitial miR-638, miR-198 and miR-146a expression in lupus nephritis. Nephrology 17:346-351

Rees F, Doherty M, Grainge MJ, Lanyon P, Zhang W (2017) The worldwide incidence and prevalence of systemic lupus erythematosus: a systematic review of epidemiological studies. Rheumatology 56:1945-1961

Reyes-Thomas J, Blanco I, Putterman C (2011) Urinary biomarkers in lupus nephritis. Clin Rev Allergy Immunol 40:138-150

Salem M, Taha H, El-Feqi MAE-F, Eesa N, Mohamed R (2018) Urinary TNF-like weak inducer of apoptosis (TWEAK) as a biomarker of lupus nephritis. Z Rheumatol 77:71-77

Schwartz N, Rubinstein T, Burkly LC, Collins CE, Blanco I, Su L, Hojaili B, Mackay M, Aranow C, Stohl W (2009) Urinary TWEAK as a biomarker of lupus nephritis: a multicenter cohort study. Arthritis Res Ther 11:1-10

Stypińska B, Paradowska-Gorycka A (2015) Cytokines and MicroRNAs as candidate biomarkers for systemic lupus erythematosus. Int J Mol Sci 16:24194-24218

Sun F, Teng J, Yu P, Li W, Chang J, Xu H (2018) Involvement of TWEAK and the NF- $\kappa \mathrm{B}$ signaling pathway in lupus nephritis. Exp Ther Med 15:2611-2619

Suttichet TB, Kittanamongkolchai W, Phromjeen C, Anutrakulchai S, Panaput T, Ingsathit A, Kamanamool N, Ophascharoensuk V, Sumethakul V, Avihingsanon Y (2019) Urine TWEAK level as a biomarker for early response to treatment in active lupus nephritis: a prospective multicentre study. Lupus Sci Med 6:e000298

Wu S, Wang J, Li F (2018) Dysregulation of PTEN caused by the underexpression of microRNA-130b is associated with the severity of lupus nephritis. Mol Med Rep 17:7966-7972

Xuejing Z, Jiazhen T, Jun L, Xiangqing X, Shuguang Y, Fuyou L (2012) Urinary TWEAK level as a marker of lupus nephritis activity in 46 cases. J Biomed Biotechnol

Publisher's Note Springer Nature remains neutral with regard to jurisdictional claims in published maps and institutional affiliations. 